\title{
Intra- and Inter-hemispheric Connectivity Supporting Hemispheric Specialization
}

\author{
Nathalie Tzourio-Mazoyer
}

\begin{abstract}
Hemispheric specialization (HS), or hemispheric dominance, is a nineteenth century concept that relates to the fact that a given hemisphere is the pilot of a given function such as, for example, the left hemisphere is dominant for language and for right-handedness. HS is grounded in both intra-hemispheric white matter connections, supported by associative bundles, and inter-hemispheric connections between cortical areas located in mirrored positions (homotopic), through the corpus callosum (CC) fiber tracts. Imaging investigations have measured anatomical and/or functional asymmetry, assessing HS at the voxelwise, regional, or hemispheric level. Comparison of these simple measures obtained with functional imaging during language tasks with results from the Wada test has validated that asymmetries do size up HS and pave the way for the investigation of HS in healthy humans. Anatomical asymmetries explain only a fraction of functional variability in lateralization, likely because structural and functional asymmetries develop at different periods of life. Anatomical asymmetries appear as early as the 26th week of gestation; at birth they are identical to those of adults. In contrast, functional neuroimaging investigations have revealed that inter-hemispheric connectivity appears at birth and is leftward asymmetrical in auditory areas, whereas in highorder language areas, this inter-hemispheric connectivity slowly shifts during development to a predominant intra-hemispheric connectivity in the adult. The precise timing and neural basis of this shift are still unknown, but it has been nevertheless shown that the connectivity is not yet in place at the age of seven and that it parallels an increase in leftward asymmetry during language tasks. Abnormal development of this asymmetry is observed in severe mental illnesses that exhibit language symptoms, such as schizophrenia and autism. In addition, after a dominant hemisphere lesion, good language capacities are associated with the recovery of a leftward asymmetry during language tasks. However, neuroimaging studies have shown that HS variability for language, up to rightward dominance, exists in healthy individuals and is partly explained by both behavioral (handedness) and anatomical (i.e., brain volume, size of the left planum temporale) factors, with these factors possibly interacting with one another. Knowledge of the setting up of
\end{abstract}

\footnotetext{
N. Tzourio-Mazoyer $(\triangle)$

Institut des Maladies Neurodégénératives (IMN), CEA CNRS Université de Bordeaux, UMR 5293, GIN Team 5, Bordeaux, France

e-mail: nathalie.tzourio-mazoyer@u-bordeaux.fr 
language HS is still fractional and very little is known about right hemisphere dominance and complementary specialization of the two hemispheres. Considering the complexity of the question, progress will come from the acquisition and analysis of databases developed to answer those questions, such as the BIL\&GIN, which includes a sample of 450 healthy volunteers balanced for handedness and gender. Each participant has been characterized for cognitive abilities, anatomy, resting state connectivity and activated networks during motor, language and visuospatial tasks.

\section{Introduction}

\section{Human Dualism: Two Hands, Two Brains}

Hemispheric specialization (HS) relates to the symmetry and asymmetry of the human body and behavior, which have imprinted human thinking. Since the birth of the first cosmological religions, the most prominent behavioral asymmetry of humans, namely handedness, has embodied dualism (in the sense of opposition) and Manichaeism. In these primitive religions, when facing the morning sun, humans had darkness and cold on their left side, warmth and light on their right side, which also faced the entire course of the sun in the sky. The association of right with south and left with north is seen in various languages, such as Celtic, old French, Irish, Sanskrit, and Hebrew (Bertrand 2001). In a still lively religion such as Catholicism, one may observe that the left is the side of evil and Inferno and the right is the side of God and Heaven. Although not explicitly stated, and although left-handers are no longer constrained or persecuted in Europe or USA, these ideas are still alive. One example can be found in Charles Laughton's 1955 movie, "The Night of the Hunter," in which the devilish reverend Harry Powell asks the children he is chasing, "Would you like me to tell you the little story of right-hand/left-hand. The story of good and evil?" At this stage, the movie star Robert Mitchum has the word "LOVE" tattooed on his right hand fingers and "HATE" on his left ones. Such an embodiment of dualism is present not only at the cultural level but also at the individual level. A recent psychological investigation demonstrated that righthanders place "good" things (namely animals in the referenced experiment) on their right, whereas left-handers do the opposite (Casasanto and Henetz 2012).

At level of the brain, handedness, one of the most lateralized behavior in humans, is related to the fact that one hemisphere-the left in right-handers-is dominant for hand control, a feature characteristic of HS. Although Max Dax and his son first conceptualized HS (Manning and Thomas-Antérion 2011), early on Broca associated the occurrence of aphasia after a left hemispheric lesion with the high prevalence of right-handedness in humans. The left hemisphere-hosting both right-handedness and language control—was declared "dominant" or "major," as opposed to the right hemisphere, which was considered as "minor." Later neuropsychological studies confirmed that, in most humans, a lesion of the left 
hemisphere leads to aphasia and apraxia whereas a lesion on the right leads to spatial neglect, attesting right hemisphere dominance for attention and visuospatial processes.

Major input to the role of each hemisphere came from split-brain investigations that have revealed the existence of hemisphere-dedicated functions, which have been demonstrated when the two hemispheres are disconnected. This approach has demonstrated the crucial role of inter-hemispheric connectivity in the setting up of HS [review in Gazzaniga (2000)]. A Manichaeism view of the hemispheres' role and function emerged in the 1980's, with a "cold" left brain hosting language and logic versus an "emotional" and creative right brain, a view that is still present in current thinking, as evidenced, for example, by recent advertising staging pictures of hemispheres with strongly contrasting characteristics.

\section{Asymmetries Measured with Brain Imaging}

The advent of functional neuroimaging has permitted the investigation of HS in healthy subjects; the first step has been to compare functional imaging results with those of Wada testing. Because the Wada procedure consists of testing language functions after anesthesia in one hemisphere, the first imaging approach designed for classifying individuals in terms of their language-dominant hemisphere has been to compute left minus right differences of activations during various language tasks, and then to categorize individuals according to an asymmetry index (positive corresponding to left-hemisphere dominance, negative to atypical individuals). Whatever the language tasks used or the technology (fCTD, fMRI) or the methodology (hemispheric, regional) applied to the computation of this asymmetry index, very consistent results have been obtained when comparing such an index with Wada testing in the same patients (Dym et al. 2011). Such validation paves the way for the use of functional imaging to investigate, through the study of interindividual variability, the factors at stake in the setting up of HS.

It is remarkable that the search for the underpinnings of hemispheric specificity, in terms of anatomical as well as functional investigations, has mainly relied on a very simple model: the calculation of a left minus right value. The implicit model underlying such a computation is that of an elementary network composed of pairs of areas, most generally located in mirrored locations, and thus principally grounded on callosal connections. As we will see, tackling differences in intrahemispheric organization has occurred more recently and was first addressed with anatomical imaging using a connectomics approach after virtually removing the corpus callosum connections (Iturria-Medina et al. 2011).

In this chapter, we will first present the current knowledge and hypotheses regarding the anatomical and functional support of HS. Second, we will discuss the consequences of recent literature describing the developmental time course of anatomical and functional inter-hemispheric organization. Third, we will present results and hypotheses regarding the relationships between HS, cognitive abilities 
and developmental/psychiatric illness. Lastly, we will comment on a tool we have designed to study HS in healthy humans, the BIL\&GIN database, with the aim of investigating the lateralization of motor, verbal, and visuospatial functions.

\section{Anatomo-Functional Support of HS}

\section{Gray Matter Macroscopic Asymmetries and HS}

The search for relationships between anatomical asymmetries in the brain and HS for language was revolutionized in 1968 when Geschwind and Levitsky (1968) reported on a leftward asymmetry of a temporal cortex area involved in speech sound processing, namely, the planum temporale (PT). This seminal finding, which was obtained through measurements of the PT surface area in post-mortem brains, was considered as a proof of an anatomical substrate for left hemisphere dominance for language. Cytoarchitectonic studies have further refined our knowledge by showing that the asymmetric PT cortex corresponds to the Tpt area, which hosts the unimodal associative auditory cortex (Galaburda et al. 1978). PT area measurements in healthy subjects using modern neuroimaging techniques started in the 1980's (Steinmetz et al. 1989), confirming that right-handers have a large leftward PT asymmetry [for a review, see Shapleske et al. (1999)].

PT asymmetry is related to a global brain torsion, named the brain Yakovlevian torque (Barrick et al. 2005), which leads to a protrusion of the right inferior frontal gyrus and of the left occipital areas [review in Toga and Thompson (2003)]. Such a torsion is also observed in $25 \%$ of great apes but its occurrence rises to more than $80 \%$ in modern humans. This asymmetrical torsion is associated with a backward shift of the left hemisphere temporal sulci, leaving a larger space at the surface of the left Sylvian fissure that hosts the PT (Lyttelton et al. 2009).

Because of the reduced occurrence of left hemisphere dominance for language in left-handers (Hécaen et al. 1981), a way to test the relationship between PT asymmetry and HS for language has been to search for differences in asymmetry between right- and left-handers. While Steinmetz reported lower PT asymmetry in left-handers (Steinmetz et al. 1991), others did not find such a difference (Habib et al. 1991; Foundas et al. 1995). This discrepancy is likely due to the fact that PT is a highly variable structure, as shown by probabilistic mapping revealing that only one voxel is common to $60 \%$ of individuals after brain normalization in the stereotaxic space (Westbury et al. 1999). This huge variability calls for large sample investigations, and we have recently reported in a sample of 273 healthy subjects that manual preference has no effect on left PT surface area or asymmetry (Tzourio-Mazoyer et al. 2010b).

Another anatomical marker of language lateralization is Heschl's gyrus, hosting the primary auditory cortex. In the left hemisphere, the size of this area is related to the volume of activated cortex during temporal processing of language sound, a 
mandatory component of language understanding (Warrier et al. 2009). Such a relationship between Heschl's structural asymmetry, or more precisely its pattern of duplication, and its functional asymmetry during speech listening has been recently reported in a large sample of healthy volunteers (Tzourio-Mazoyer et al. 2015).

In the frontal lobe, results concerning an asymmetry of the inferior frontal gyrus, hosting Broca's area in the left hemisphere, have been inconsistent, but a recent report in a large sample of 200 healthy adults showed a leftward asymmetry of the insula that was related to word recognition lateralization (Chiarello et al. 2013). Moreover, this work revealed that the anatomo-functional relationship between these asymmetries was stronger than the one observed with the PT, demonstrating that the search for anatomical markers of HS is still lively.

However, one should keep in mind that, even if anatomical and functional asymmetries during language tasks can be found to be correlated, such a relationship must be quite weak, considering that some studies found evidence for it (Tzourio et al. 1998; Josse et al. 2006, 2009) whereas others did not (Eckert et al. 2006). As will be further developed, this discrepancy is likely due to a stronger correspondence existing in primary areas having an early anatomical and functional maturation than in high-order cortices that show a delayed anatomical and functional development (Hill et al. 2010b), leaving environmental factors to exert a stronger influence.

\section{White Matter Connections Supporting HS}

In terms of White Matter (WM) anatomy, HS is grounded both in intra-hemispheric connections supported by associative bundles and in inter-hemispheric connections between cortical areas located in mirrored positions (homotopic) connected by the corpus callosum [review in van der Knaap and van der Ham (2011)].

\section{Corpus Callosum}

The two hemispheres are connected by the CC, which is made up of 200 million fibers issuing from pyramidal cells of cortical layers II and III with homotopic projections on contralateral neurons of the same layer. Major advances in defining the functional role of the $\mathrm{CC}$ have come from investigations of split-brain patients with partial or total hemispheric disconnection after callosotomy. Cases of partial callosotomy have shown that CC is topographically organized, with transfer of visual, auditory and somatosensory information in its posterior parts and of attentional resources and higher cognitive information in more anterior regions. Investigations conducted by Sperry and Gazzaniga established the allocation of functions between the two hemispheres and the fundamental role of $\mathrm{CC}$ in the transfer of information between them [review in Gazzaniga (2000)]. They also described the alien-hand syndrome and conflicting hand-motor behavior in the acute phase after 
$\mathrm{CC}$ surgery, demonstrating that the $\mathrm{CC}$ also has an inhibitory role that is crucial for human behavior. The $\mathrm{CC}$ has thus been at center stage in the investigation of HS anatomical support.

Considering that, in the course of evolution, there has been a decrease in CC size with increasing brain size and complexity, it is assumed that a smaller CC was associated with increased hemispheric lateralization of functions (Hopkins and Cantalupo 2008). In humans, it has also been shown that large brains have relatively smaller CC surface area (Jäncke and Steinmetz 1998); these authors considered that this finding supported the theory proposed by Ringo that increasing size, and thereby inter-hemispheric transfer, was a mechanical factor favoring the grouping of areas supporting a given function within one hemisphere (Ringo et al. 1994). However, there is also evidence of increased hemispheric lateralization associated with increased CC size, at least in some of its subparts. For example, in 74 healthy volunteers in whom language lateralization was measured during a semantic decision task on written words, a stronger lateralization was associated with increased mid-sagittal CC size (Josse et al. 2008). These apparent discrepancies in the literature are likely due to several difficulties in $\mathrm{CC}$ functional exploration. First, $\mathrm{CC}$ contains both small diameter fibers conveying inhibitory connections across high-order areas and large fast-conducting fibers connecting primary and unimodal associative areas; it is thus difficult to infer its role only from anatomical variables. Second, the topographical organization of CC is complex and fine-grained, and until now, most investigations have mainly relied on the total surface area or on a coarse parcellation scheme. A detailed mapping of the topographical organization of CC based on DTI data is ongoing in healthy volunteers, and its findings will be very useful for future research (Putnam et al. 2010; Chao et al. 2009).

\section{Intra-hemispheric Structural Connectivity}

The two hemispheres appear to have a very comparable anatomical organization in terms of WM fiber bundles. The coarse picture that one gets from long-distance fasciculus organization in the brain is that of a globally symmetrical pattern, in accordance with the fact that the right and left hemispheres have comparable organization in terms of cortical hierarchy and cognitive network organization (Mesulam 2000; Fuster 2009). Only a few investigations have reported WM asymmetries, and all focused on the arcuate fasciculus, which is known to support language on the left, and on the cortico-spinal tract that connects the motoneurons to the medulla. Using DTI, the initial observation of bilateral arcuate fasciculus, with subjects without a right arcuate fasciculus (Catani et al. 2007), has not been replicated, although a leftward asymmetry of the arcuate fasciculus and corticospinal tracts seems present in healthy adults (Thiebaut de Schotten et al. 2011). A leftward asymmetry in terms of fractional anisotropy has also been observed in the WM connecting frontal and occipital areas (Suchan et al. 2013).

The picture is clearer in newborns, where leftward asymmetry of the arcuate fasciculus has been observed at birth (Dubois et al. 2009; Leroy et al. 2011). Thus, 
even if hemispheric dominance corresponds to differences in cognitive processes, the structure of the two hemispheres follows the same anatomo-functional organizational rules. Differences are thus likely to be subtle.

Even though investigating differences in WM intra-hemispheric organization may seem complex, Iturria-Medina et al. (2011) have developed a promising approach, applying graph analyses to WM images obtained with DTI. The originality of this approach is that WM hemisphere graphs are computed after a virtual cut of the inter-hemispheric callosal connections, making it possible to unravel potential differences that were probably masked by the strength of existing callosal connections. Although the results were obtained in a limited sample of individuals, they evidenced hemispheric differences: the left hemisphere was hosting more nodes, whereas the right had more connections. Comparison of this observation to functional or behavioral observations will allow us to progress in understanding these hemispheric differences in WM connectivity.

\section{Functional Asymmetries and Hemispheric Dominance for Language Assessed with Meta-analysis}

Language was one of the first cognitive functions scrutinized with functional imaging (Petersen et al. 1988). Since its advent, functional imaging has developed tools allowing for the averaging of different brains in a common reference system (Fox et al. 1985). This approach allowed for conducting a posteriori meta-analyses that permitted a precise description of the localization of brain areas activated during various tasks. Using the localization of activated regions as the starting point, it is possible to speculate about the role of these regions by analyzing their involvement in different tasks.

Using a meta-analysis approach, we evaluated the relative roles of the left and right hemispheres during linguistic tasks by analyzing 128 functional imaging studies dealing with language tasks in healthy, right-handed participants (Vigneau et al. 2006). We found 59 articles reporting right-hemisphere participation, with 105 language contrasts providing 218 peaks that were analyzed in a second step (Vigneau et al. 2011). Compared to the 728 peaks observed in the left hemisphere, the low proportion of right-hemisphere participation in the same studies was early evidence of the left-hemisphere language dominance. To better characterize hemispheric participation, we described inter-hemispheric interactions in each of the language contrasts involving both hemispheres. We classified peaks as unilateral or bilateral. During a given task of a given study, a unilateral peak should not exhibit any homotopic activation, homotopic activation being defined as the presence of an activation focus located in a mirror position in the other hemisphere. In contrast, a bilateral peak was defined when it was accompanied by homotopic activation in the opposite hemisphere during the same contrast. 
We computed the proportion of unilateral and bilateral peaks in each hemisphere and observed that, while the majority of left hemisphere peaks were unilateral (79 \%), a reverse pattern was observed on the right (67\% bilateral). These results demonstrated that the left hemisphere works in an intra-hemispheric manner in adults, in contrast to the right hemisphere, which is under the dominance of the left hemisphere (Vigneau et al. 2011). As developed below, this observation accords with recent investigations of intrinsic connectivity (Perani et al. 2011) and functional connectivity during sentence listening (Friederici 2011), which demonstrated with a seed approach the existence of strong intra-hemispheric temporal connectivity across frontal and temporal high-order language areas.

\section{Developmental Course of the Setting Up of Anatomical and Functional Asymmetries}

Anatomical asymmetries, in terms of depth of the Sylvian fissure, have been observed with MRI as early as the 26th week of gestation (Habas et al. 2012), and such an asymmetry is at birth identical to that of adults (Hill et al. 2010a). It is important to underline that later studies have shown that these asymmetries do not evolve much during childhood ( $\mathrm{Li}$ et al. 2013). On the functional side, in utero, neuroimaging investigations have revealed that intrinsic connectivity remains local until birth, when inter-hemispheric connectivity appears (Smyser et al. 2011). Applying a seed approach, Perani et al. (2011) specifically investigated the intrinsic connectivity of the left inferior frontal gyrus and the left superior temporal gyrus, two areas where language develops. They showed that that, at birth, these regions were only connected to homotopic areas, with no intra-hemispheric intrinsic connectivity. This birth pattern was opposed to that of adults included in the same study, who exhibited a preeminent left intra-hemispheric synchronization of BOLD variation at rest in this fronto-temporal network (Perani et al. 2011). However, one should note that, in primary auditory cortices that exhibit a leftward anatomical asymmetry at birth ( $\mathrm{Li}$ et al. 2013), a leftward functional asymmetry is present when infants listen to language, whereas a rightward one is revealed when they listen to music (Dehaene-Lambertz et al. 2010). This latter study demonstrates a consistency between anatomical and functional asymmetries of the Sylvian fissure cortex at birth and that the inter-hemispheric connectivity is a key element of the development of the lateralization of auditory cortices. However, the change in the organization of higher-order language areas from an initial inter- to the intrahemispheric organization of adults is still not in place at 7 years of age (Friederici et al. 2011). In this study, 5-7-year-old children were presented with four conditions: two including correct sentences, one including semantically incorrect sentences, and one including syntactically incorrect sentences. The children's task was to judge the acceptability of the sentences. Applying a seed in areas that had been identified in functional MRI studies as supporting sentence processing 
[namely, the left dorsal inferior frontal gyrus (IFG) and the left posterior superior temporal gyrus (STG) and sulcus (STS)], the authors showed that, when seeded in the left posterior STS, strong correlations with the left IFG were found in adults. For children, in contrast to adults, the analysis revealed strong correlations with the contralateral temporal region. The same observation was present when the seed was in the IFG. Within the same period in which homotopic connections prevail, a 5 -year longitudinal study showed a linear increase with age of the left hemisphere involvement in the IFG during verb generation (Szaflarski et al. 2006), an additional demonstration that language left-hemisphere specialization develops first through callosal interactions.

As a whole, these recent functional imaging studies show that anatomical and functional asymmetries of auditory primary areas are in place at birth, whereas in high-order language areas (IFG, STS, STG) leftward asymmetries develop slowly along with verbal acquisition, before reaching the adult pattern of a dominant intrahemispheric processing of language. To our knowledge, the exact time course and the physiological underpinnings of this developmental switch from inter- to intrahemispheric functioning during language processing remain to be established. It is not known whether this type of developmental scenario is also at stake for other left-lateralized function, such as praxis (Vingerhoets et al. 2013), or for rightlateralized functions, such as spatial attention.

\section{HS, Cognitive Abilities and Developmental/Psychiatric Diseases}

\section{Cognitive Skills and Asymmetries}

How does this developmental change in brain organization relate to cognitive development and abilities? Everts et al. (2009) mapped 9-21-year-old healthy participants during rhyming and synonym language tasks and measured both their hemispheric asymmetry and their verbal abilities. They observed that the increase in leftward asymmetry was linearly correlated with age but also, independently of age, with verbal performances. Importantly, in the same individuals they measured hemispheric asymmetries during a visuospatial task and observed the reverse pattern for this right hemisphere-dominant function: a right asymmetry increase with age, and the larger the rightward asymmetry, the better the visuospatial performances of the participants (Everts et al. 2009).

As will be further developed, the case is not so clear in healthy adults, but there is a study pointing toward an association between leftward asymmetry and verbal performances in individuals who had suffered from pre- or perinatal stroke. Twenty-five of such subjects were mapped (7-23 years old) during a word generation task and a measure of asymmetry in their IFG activity during this task was computed. As opposed to a control group, they did not show a leftward asymmetry 
because of the recruitment of the right IFG during the language task, which was considered as a compensatory or plastic participation. Importantly, the analysis of the relationship between verbal abilities of these patients and their laterality index evidenced that, the more leftward the asymmetry, the better the performances, showing that a good recovery was associated with the possibility of regaining a left-hemisphere dominance for language (Raja Beharelle et al. 2010).

As reviewed by Cathy Price (Price and Crinion 2005), the role of interhemispheric connections is essential and complex during the recovery of production aphasia after a stroke. The quality of recovery depends on slowly evolving activation changes in the left hemisphere. By contrast, right hemisphere activation observed after a left hemisphere lesion has been interpreted as the consequence of a transcallosal dis-inhibition that is not directly involved in recovery. Such activations occur early after stroke in areas homotopic to the lesion site, and their intensity does not correlate with the level of recovery, as opposed to that of peri-lesional activations (Rosen et al. 2000; Perani et al. 2003). Note that, unlike speech production, recovery of speech comprehension appears to depend on both left and right temporal lobe activation (Price and Crinion 2005).

Many investigations of the relationships between cognitive skills and brain asymmetries in healthy adults have been conducted through a comparison between right- and left-handers, whereas very few directly tested a relationship between hemispheric functional lateralization and cognitive performances. Based on the divided visual field paradigm, a series of behavioral studies in healthy participants have addressed the issue of the benefit of hemispheric lateralization (Boles et al. 2008; Chiarello et al. 2009; Hirnstein et al. 2010). In this paradigm, difference in performances following a presentation of the stimuli in either the left hemi-field (right hemisphere) or the right hemi-field (left hemisphere) is interpreted as an index reflecting the hemispheric dominance for the stimuli processing. These studies have reported divergent outcomes, some emphasizing a positive correlation between the index of lateralization for various linguistic tasks and reading skills (Chiarello et al. 2009) whereas other reported that high degrees of lateralization were detrimental to cognitive performance in word-matching and face-decision tasks (Hirnstein et al. 2010). A study that used functional transcranial Dopler sonography (fTCD) to directly assess the hemispheric dominance did not report any relationship between hemispheric asymmetry and the number of foreign languages spoken fluently, academic achievement and the practice of artistic activities or, in a sub-group of 21 participants, general IQ (Knecht et al. 2001). A recent study including 6-24-year-old right-handed participants revealed differences according to the language task used for measuring asymmetries. While there was a correlation between verbal IQ and the hemispheric functional lateralization index obtained by fMRI during language comprehension, the correlation was absent between verbal IQ and hemispheric asymmetries during language production. In addition, better performances were associated with larger right hemisphere participation (Lidzba et al. 2011).

In summary, there is an association between verbal abilities and a leftward hemispheric asymmetry during a language task in the developmental course. 
Leftward asymmetry during language production is also associated with a good recovery of production aphasia. However, there is no such evidence of an association between language skills and leftward hemispheric lateralization in healthy adults, although the limited number of investigations leaves the question open. Moreover, one should tackle the issue of whether such an association is specific to language skills rather than to more general cognitive functioning abilities.

\section{Language HS, Developmental and Psychiatric Diseases}

Discovery of the PT leftward asymmetry triggered the search for an association between a decrease in this asymmetry and the occurrence of developmental language disorders such as dysphasia and dyslexia. Geschwind and Galaburda (1985a, b) have elaborated a model of the setting up of left hemisphere dominance based on the idea that asymmetries are due to a reduction of right-hemispheric structures through development. Their model linked developmental language pathologies to decreases in anatomical asymmetries. There have been numerous works showing an association between language developmental pathologies and language lateralization markers, in particular decreases in anatomical asymmetries [reviewed in Leonard and Eckert (2008)]. Such differences in language asymmetry in developmental pathologies are modest but have been associated with other severe pathologies encompassing language deficits such as schizophrenia and autism.

Failure to develop normal language comprehension is an early sign of autism and, in this developmental pathology, a deficit in the setting up of a language leftward functional asymmetry has also been reported by Eyler et al. (2012), who used fMRI to investigate hemispheric asymmetries in 12-48-month-old toddlers with autistic spectrum disorder who were later diagnosed with autism. Measuring their brain activity during story listening while toddlers were asleep, they observed not only a decreased leftward lateralization in the temporal cortex as compared to control children but also a trend to a rightward temporal increase in activation between 1 and 4 years of age, as opposed to the typical increase in leftward asymmetry in normally developing children. The authors interpreted their findings as attesting that a failure in the setting up of leftward asymmetry during language processing is a fundamental abnormality of autism (Eyler et al. 2012).

Decreases in the anatomical and functional asymmetry of language areas have also been reported in schizophrenia. Starting from the saliency of language defects in schizophrenic patients, Crow (1997) proposed that schizophrenia could be considered an anomaly of the function of language and that the pathophysiology of schizophrenia should be found in the mechanisms underlying the development of HS. Crow's hypothesis was based on the observation that pre-schizophrenic children were more likely to be rated as ambidextrous at the age of 7 years and were less strongly right-handed than their peers at the age of 11, suggesting a delay in the establishment of their HS (Crow et al. 1996). In addition, left hemisphere anatomical abnormalities centered on the temporal lobe were reported in schizophrenic 
patients with a decreased asymmetry of the PT (DeLisi 1997; Sommer et al. 2001). In an fMRI investigation of right-handed schizophrenic patients during a language comprehension task, Dollfus et al. (2005) observed decreased left hemispheric activity in language regions in patients as compared to matched healthy controls. Such a finding was also reported in the first episode of schizophrenic patients during a language generation task, making the decrease in asymmetry independent of the type of language task and of the potential effects of the illness and its treatment (Bleich-Cohen et al. 2009). In addition, it was shown that the decreased leftward asymmetry during verbal production in schizophrenic patients was not related to auditory hallucinations (Diederen et al. 2010).

As a whole, a defect in the setting up of language leftward asymmetry is observed in severe mental pathologies that include language dysfunction among other symptoms. The deficit in leftward lateralization targets a dysfunction of HS as an early developmental mechanism fundamental to further harmonious development of functional brain architecture. Further research is needed to evaluate whether this developmental failure results in a pure language lateralization deficit or corresponds to a general dysfunction in the lateralization of cognitive functions.

\section{Inter-individual Variability in HS: Factors at Play}

Although encountered in pathological conditions, a decrease in language lateralization can also be found in healthy individuals, as revealed by neuroimaging studies describing between-individual variability of hemispheric or regional functional lateralization of language. As developed below, variability in language lateralization of healthy individuals is multifactorial and depends on both behavioral characteristics, such as handedness, and anatomical features, such as brain volume or size of the left PT. Variability in functional lateralization also depends on the type of language process targeted by the language task performed during imaging, language production being more strongly leftward lateralized than language comprehension. Finally, it must be underscored that language lateralization varies according to the hierarchical level of the regions studied: primary areas receiving bilateral sensory inputs have a lateralization that is weaker than that of high-order language areas, and factors explaining this regional variability may be different. For example, the pattern of gyrification of Heschl's gyrus explains the variability in asymmetry of this region during speech listening but not that of other areas activated during this task, such as the STS (Tzourio-Mazoyer et al. 2015).

Handedness was identified early on as a source of between-subject variability in language lateralization. The fact that more than $90 \%$ of right-handers have a left dominance for language has nourished both evolutionary and genetic models of the origin of language [reviewed in Corballis et al. (2012)]. However, it must be stressed that around $80 \%$ of left-handers exhibit the same typical left lateralization during language production and that the increased variability of language lateralization within left-handers is characterized by the existence of rare rightward 
asymmetrical individuals, who in addition exhibit strong left-hand preference (Pujol et al. 1999; Knecht et al. 2000). Note, however, that apart from these rare individuals having reverse language lateralization, occurring with similar proportions in children and adults, handedness has no influence on the maturational increase of leftward asymmetries for language (Szaflarski et al. 2011).

The fact that dissociations have been observed between the lateralization of language areas involved during language production and those involved in language perception suggests that there are different factors related to specific aspects of speech processing. In favor of this hypothesis is the evidence that anatomical factors explain a part of the variability of anatomical or functional lateralization of speech processing areas. Among them, brain volume determines inter-hemispheric distance and transfer time. According to Ringo et al. (1994), brain volume constrains high-speed processes to intra-hemispheric clustering in bulky brains. This theory fits within the framework of perceptual theories of the origin of language lateralization postulating that it arises from speed constraints on speech perception. As a matter of fact, we found positive correlations between brain volume and both leftward functional asymmetry during speech perception (Josse et al. 2006; Tzourio-Mazoyer et al. 2010a) and leftward gray matter hemispheric asymmetry (Tzourio-Mazoyer et al. 2010b). These results support Ringo's theory of a 'mechanical' impact of brain volume on speech lateralization. Other arguments come from the fact that anatomical characteristics of the auditory cortices explain a part of the functional variability in language lateralization, as, for example, the positive correlation between the left PT surface area and lateralization of activations during story listening (Tzourio et al. 1998; Josse et al. 2003).

The picture is likely to be even more complex, given that factors can interact. For example, in right-handers, we showed that weaker manual lateralization decreases leftward lateralization for language only in individuals with familial sinistrality (Tzourio-Mazoyer et al. 2010a). Moreover, factors that influence variability in HS, such as gender and brain volume, may also be partially confounded (Leonard et al. 2008). Finally, it must be emphasized that, although much is known about HS for language, the factors that might influence right hemisphere specialization remain to be discovered.

\section{BIL\&GIN: A Multimodal Database for Investigating HS}

To address some of the issues raised in this chapter and to make progress in our understanding of the role of HS in shaping the large-scale organization of the human brain, we acquired a multimodal (neuroimaging, cognitive/behavioral abilities, genetic) database designed for the investigation of HS. This database, named BIL\&GIN (Brain Imaging of Lateralization by the Groupe d'Imagerie Neurofonctionnelle), included a sample of 453 healthy adults (aged 18-54 years), balanced for sex and handedness (Mazoyer et al. 2015). For each participant, we recorded manual skills, hand and eye preference, and familial sinistrality. Verbal, spatial, 
and numerical abilities were assessed with a large battery of tests. Finally, multimodal MRI data were acquired in each participant, namely T1 and DTI for conducting morphometric analysis of gray matter and WM, and resting-state fMRI data for assessing intrinsic connectivity. Finally, in a subsample of 300 individuals, task-related fMRI was performed using a battery of 15 language, motor and visuospatial tasks designed to explore various aspects of HS. The first analyses of the BIL\&GIN illustrate the power of combining a large sample with a multimodal approach. For example, we recently reported that the verbal and spatial abilities increase with right asymmetry in motor skills and that cognitive performance is reduced in participants having a familial sinistrality combined with non-maximal preference strength of the dominant hand (Mellet et al. 2014). Original findings regarding the Heschl's gyrus interhemispheric duplication pattern (Marie et al. 2015) and its relationship with the functional asymmetry of this area during speech listening (Tzourio-Mazoyer et al. 2015) were also previously mentioned in this chapter.

\section{Conclusion}

HS, which is grounded mainly by inter-hemispheric connectivity, is an essential feature of human anatomo-functional brain architecture. This very simple right-left connection is essential to the development of language and, likely, to optimal cognitive functioning. We believe that its investigation within the framework of the research on the connectomics of the brain will provide important knowledge regarding the large-scale architecture supporting human cognition.

Open Access This chapter is distributed under the terms of the Creative Commons AttributionNoncommercial 2.5 License (http://creativecommons.org/licenses/by-nc/2.5/) which permits any noncommercial use, distribution, and reproduction in any medium, provided the original author(s) and source are credited.

The images or other third party material in this chapter are included in the work's Creative Commons license, unless indicated otherwise in the credit line; if such material is not included in the work's Creative Commons license and the respective action is not permitted by statutory regulation, users will need to obtain permission from the license holder to duplicate, adapt or reproduce the material.

\section{References}

Barrick TR, Mackay CE, Prima S, Maes F, Vandermeulen D, Crow TJ, Roberts N (2005) Automatic analysis of cerebral asymmetry: an exploratory study of the relationship between brain torque and planum temporale asymmetry. Neuroimage 24:678-691

Bertrand P-M (2001) Histoire des gauchers: des gens à l'envers. Imago, Paris

Bleich-Cohen M, Hendler T, Kotler M, Strous RD (2009) Reduced language lateralization in firstepisode schizophrenia: an fMRI index of functional asymmetry. Psychiatry Res 171:82-93

Boles DB, Barth JM, Merrill EC (2008) Asymmetry and performance: toward a neurodevelopmental theory. Brain Cogn 66:124-139

Casasanto D, Henetz T (2012) Handedness shapes children's abstract concepts. Cogn Sci 36:359-372 
Catani M, Allin MP, Husain M, Pugliese L, Mesulam MM, Murray RM, Jones DK (2007) Symmetries in human brain language pathways correlate with verbal recall. Proc Natl Acad Sci USA 104:17163-17168

Chao YP, Cho KH, Yeh CH, Chou KH, Chen JH, Lin CP (2009) Probabilistic topography of human corpus callosum using cytoarchitectural parcellation and high angular resolution diffusion imaging tractography. Hum Brain Mapp 30:3172-3187

Chiarello C, Welcome SE, Halderman LK, Towler S, Julagay J, Otto R, Leonard CM (2009) A large-scale investigation of lateralization in cortical anatomy and word reading: are there sex differences? Neuropsychology 23:210-222

Chiarello C, Vazquez D, Felton A, Leonard CM (2013) Structural asymmetry of anterior insula: behavioral correlates and individual differences. Brain Lang 126:109-122

Corballis MC, Badzakova-Trajkov G, Häberling IS (2012) Right hand, left brain: genetic and evolutionary bases of cerebral asymmetries for language and manual action. Wiley Interdiscip Rev Cogn Sci 3:1-17

Crow TJ (1997) Is schizophrenia the price that Homo sapiens pays for language? Schizophr Res 28:127-141

Crow TJ, Done DJ, Sacker A (1996) Cerebral lateralization is delayed in children who later develop schizophrenia. Schizophr Res 22:181-185

Dehaene-Lambertz G, Montavont A, Jobert A, Allirol L, Dubois J, Hertz-Pannier L, Dehaene S (2010) Language or music, mother or Mozart? Structural and environmental influences on infants' language networks. Brain Lang 114:53-65

DeLisi LE (1997) Anomalous cerebral asymmetry and language processing in schizophrenia. Schizophr Bull 23:536

Diederen KM, De Weijer AD, Daalman K, Blom JD, Neggers SF, Kahn RS, Sommer IE (2010) Decreased language lateralization is characteristic of psychosis, not auditory hallucinations. Brain 133:3734-3744

Dollfus S, Razafimandimby A, Delamillieure P, Brazo P, Joliot M, Mazoyer B, Tzourio-Mazoyer N (2005) Atypical hemispheric specialization for language in right-handed schizophrenia patients. Biol Psychiatry 57:1020-1028

Dubois J, Hertz-Pannier L, Cachia A, Mangin JF, Le Bihan D, Dehaene-Lambertz G (2009) Structural asymmetries in the infant language and sensori-motor networks. Cereb Cortex 19:414-423

Dym RJ, Burns J, Freeman K, Lipton ML (2011) Is functional MR imaging assessment of hemispheric language dominance as good as the Wada test?: a meta-analysis. Radiology 261:446-455

Eckert MA, Leonard CM, Possing ET, Binder JR (2006) Uncoupled leftward asymmetries for planum morphology and functional language processing. Brain Lang 98:102-111

Everts R, Lidzba K, Wilke M, Kiefer C, Mordasini M, Schroth G, Perrig W, Steinlin M (2009) Strengthening of laterality of verbal and visuospatial functions during childhood and adolescence. Hum Brain Mapp 30:473-483

Eyler LT, Pierce K, Courchesne E (2012) A failure of left temporal cortex to specialize for language is an early emerging and fundamental property of autism. Brain 135:949-960

Foundas AL, Leonard CM, Heilman KM (1995) Morphological asymmetries and handedness. The pars triangularis and planum temporale. Arch Neurol 52:501-508

Fox PT, Perlmutter JSA, Raichle ME (1985) A stereotactic method of anatomical localization for positron emission tomography. J Comput Assist Tomogr 9:141-153

Friederici AD (2011) The brain basis of language processing: from structure to function. Physiol Rev 91:1357-1392

Friederici AD, Brauer J, Lohmann G (2011) Maturation of the language network: from inter- to intrahemispheric connectivities. PLoS One 6:e20726

Fuster JM (2009) Cortex and memory: emergence of a new paradigm. J Cogn Neurosci 21:2047-2072 
Galaburda AM, LeMay M, Kemper TL, Geschwind N (1978) Right-left asymmetries in the brain. Science 199:852-856

Gazzaniga MS (2000) Cerebral specialization and interhemispheric communication-does the corpus callosum enable the human condition? Brain 123:1293-1326

Geschwind N, Galaburda AM (1985a) Cerebral lateralization. Biological mechanisms, associations, and pathology:1. A hypothesis and a programm for research. Arch Neurol 42:428-459

Geschwind N, Galaburda AM (1985b) Cerebral lateralization. Biological mechanisms, associations, and pathology: 2. A hypothesis and a programm for research. Arch Neurol 42:521-552

Geschwind N, Levitsky W (1968) Human brain left-right asymmetries in temporal speech region. Science 161:186-187

Habas PA, Scott JA, Roosta A, Rajagopalan V, Kim K, Rousseau F, Barkovich AJ, Glenn OA, Studholme C (2012) Early folding patterns and asymmetries of the normal human brain detected from in utero MRI. Cereb Cortex 22:13-25

Habib M, Gayraud D, Oliva A, Regis J, Salamon G, Khalil R (1991) Effects of handedness and sex on the morphology of the corpus callosum: a study with brain magnetic resonance imaging. Brain Cogn 16:41-61

Hécaen H, De Agostini M, Monzon-Montes A (1981) Cerebral organization in left-handers. Brain Lang 12:261-284

Hill J, Dierker D, Neil J, Inder T, Knutsen A, Harwell J, Coalson T, Van Essen D (2010a) A surface-based analysis of hemispheric asymmetries and folding of cerebral cortex in term-born human infants. J Neurosci 30:2268-2276

Hill J, Inder T, Neil J, Dierker D, Harwell J, Van Essen D (2010b) Similar patterns of cortical expansion during human development and evolution. Proc Natl Acad Sci USA 107:13135-13140

Hirnstein M, Leask S, Rose J, Hausmann M (2010) Disentangling the relationship between hemispheric asymmetry and cognitive performance. Brain Cogn 73:119-127

Hopkins WD, Cantalupo C (2008) Theoretical speculations on the evolutionary origins of hemispheric specialization. Curr Dir Psychol Sci 17:233-237

Iturria-Medina Y, Pérez Fernández A, Morris DM, Canales-Rodríguez EJ, Haroon HA, García Pentón L, Augath M, Galán García L, Logothetis N, Parker GJ, Melie-García L (2011) Brain hemispheric structural efficiency and interconnectivity rightward asymmetry in human and nonhuman primates. Cereb Cortex 21:56-67

Jäncke L, Steinmetz H (1998) Brain size: a possible source of interindividual variability in corpus callosum morphology. In: Zaidel E, Iacoboni M, Pascual-Leone A (eds) Brain size: a possible source of interindividual variability in corpus callosum morphology. Plenum Press, New York, pp $1-15$

Josse G, Mazoyer B, Crivello F, Tzourio-Mazoyer N (2003) Left planum temporale: an anatomical marker of left hemispheric specialization for language comprehension. Brain Res Cogn Brain Res 18:1-14

Josse G, Hervé PY, Crivello F, Mazoyer B, Tzourio-Mazoyer N (2006) Hemispheric specialization for language: brain volume matters. Brain Res 1068:184-193

Josse G, Seghier ML, Kherif F, Price CJ (2008) Explaining function with anatomy: language lateralization and corpus callosum size. J Neurosci 28:14132-14139

Josse G, Kherif F, Flandin G, Seghier ML, Price CJ (2009) Predicting language lateralization from gray matter. J Neurosci 29:13516-13523

Knecht S, Dräger B, Deppe M, Bobe L, Lohmann H, Flöel A, Ringelstein EB, Henningsen H (2000) Handedness and hemispheric language dominance in healthy humans. Brain 123:2512-2518

Knecht S, Drager B, Floel A, Lohmann H, Breitenstein C, Deppe M, Henningsen H, Ringelstein EB (2001) Behavioural relevance of atypical language lateralization in healthy subjects. Brain 124:1657-1665

Leonard CM, Eckert MA (2008) Asymmetry and dyslexia. Dev Neuropsychol 33:663-681 
Leonard CM, Towler S, Welcome S, Halderman LK, Otto R, Eckert MA, Chiarello C (2008) Size matters: cerebral volume influences sex differences in neuroanatomy. Cereb Cortex 18:2920-2931

Leroy F, Glasel H, Dubois J, Hertz-Pannier L, Thirion B, Mangin JF, Dehaene-Lambertz G (2011) Early maturation of the linguistic dorsal pathway in human infants. J Neurosci 31:1500-1506

Li G, Nie J, Wang L, Shi F, Lyall AE, Lin W, Gilmore JH, Shen D (2013) Mapping longitudinal hemispheric structural asymmetries of the human cerebral cortex from birth to 2 years of age. Cereb Cortex 24:1289-1300

Lidzba K, Schwilling E, Grodd W, Krägeloh-Mann I, Wilke M (2011) Language comprehension vs. language production: age effects on fMRI activation. Brain Lang 119:6-15

Lyttelton OC, Karama S, Ad-Dab'bagh Y, Zatorre RJ, Carbonell F, Worsley K, Evans AC (2009) Positional and surface area asymmetry of the human cerebral cortex. Neuroimage 46:895-903

Manning L, Thomas-Antérion C (2011) Marc Dax and the discovery of the lateralisation of language in the left cerebral hemisphere. Rev Neurol (Paris) 167:868-872

Marie D, Jobard G, Crivello F, Perchey G, Petit L, Mellet E, Joliot M, Zago L, Mazoyer B, Tzourio-Mazoyer N (2015) Descriptive anatomy of Heschl's gyri in 430 healthy volunteers, including 198 left-handers. Brain Struct Funct 220:729-743

Mazoyer B, Mellet E, Perchey G, Zago L, Crivello F, Jobard G, Delcroix N, Vigneau M, Leroux G, Petit L, Joliot M, Tzourio-Mazoyer N (2015) BIL\&GIN: a neuroimaging, cognitive, behavioral, and genetic database for the study of human brain lateralization. Neuroimage 124: $1225-1231$

Mellet E, Jobard G, Zago L, Crivello F, Petit L, Joliot M, Mazoyer B, Tzourio-Mazoyer N (2014) Relationships between hand laterality and verbal and spatial skills in 436 healthy adults balanced for handedness. Laterality 2014(19):383-404

Mesulam MM (2000) In: Mesulam MM (ed) Principles of behavioral neurology. Oxford University Press, Oxford

Perani D, Cappa SF, Tettamanti M, Rosa M, Scifo P, Miozzo A, Basso A, Fazio F (2003) A fMRI study of word retrieval in aphasia. Brain Lang 85:357-368

Perani D, Saccuman MC, Scifo P, Anwander A, Awander A, Spada D, Baldoli C, Poloniato A, Lohmann G, Friederici AD (2011) Neural language networks at birth. Proc Natl Acad Sci USA 108:16056-16061

Petersen SE, Fox PT, Posner MI, Mintun MA, Raichle ME (1988) Positron emission tomographic studies of the cortical anatomy of single-word processing. Nature 331:585-589

Price CJ, Crinion J (2005) The latest on functional imaging studies of aphasic stroke. Curr Opin Neurol 18:429-434

Pujol J, Deus J, Losilla JM, Capdevela A (1999) Cerebral lateralization of language in normal lefthanded people studied by functional MRI. Neurology 52:1038-1043

Putnam MC, Steven MS, Doron KW, Riggall AC, Gazzaniga MS (2010) Cortical projection topography of the human splenium: hemispheric asymmetry and individual differences. J Cogn Neurosci 22:1662-1669

Raja Beharelle A, Dick AS, Josse G, Solodkin A, Huttenlocher PR, Levine SC, Small SL (2010) Left hemisphere regions are critical for language in the face of early left focal brain injury. Brain 133:1707-1716

Ringo JL, Doty RW, Demeter S, Simard PY (1994) Time is of the essence: a conjecture that hemispheric specialization arises from interhemispheric conduction delay. Cereb Cortex 4:331-343

Rosen HJ, Petersen SE, Linenweber MR, Snyder AZ, White DA, Chapman L, Dromerick AW, Fiez JA, Corbetta M (2000) Neural correlates of recovery from aphasia after damage to left inferior frontal cortex. Neurology 55:1883-1894

Shapleske J, Rossell SL, Woodruff PW, David AS (1999) The planum temporale: a systematic, quantitative review of its structural, functional and clinical significance. Brain Res Rev 29:26-49 
Smyser CD, Snyder AZ, Neil JJ (2011) Functional connectivity MRI in infants: exploration of the functional organization of the developing brain. Neuroimage 56:1437-1452

Sommer IEC, Ramsey NF, Kahn RS (2001) Language lateralization in schizophrenia, an fMRI study. Schizophr Res 52:57-67

Steinmetz H, Rademacher J, Huang Y, Hefter H, Zilles K, Thron A, Freund H (1989) Cerebral asymmetry: MR planimetry of the human planum temporale. J Comput Assist Tomogr 13:996-1005

Steinmetz H, Volkmann J, Jäncke L, Freund H (1991) Anatomical left-right asymmetry of language-related temporal cortex is different in left- and right-handers. Ann Neurol 29:315-319

Suchan J, Umarova R, Schnell S, Himmelbach M, Weiller C, Karnath HO, Saur D (2013) Fiber pathways connecting cortical areas relevant for spatial orienting and exploration. Hum Brain Mapp 35:1031-1043

Szaflarski JP, Holland SK, Schmithorst VJ, Byars AW (2006) fMRI study of language lateralization in children and adults. Hum Brain Mapp 27:202-212

Szaflarski JP, Rajagopal A, Altaye M, Byars AW, Jacola L, Schmithorst VJ, Schapiro MB, Plante E, Holland SK (2011) Left-handedness and language lateralization in children. Brain Res 1433:85-97

Thiebaut de Schotten M, Ffytche DH, Bizzi A, Dell'Acqua F, Allin M, Walshe M, Murray R, Williams SC, Murphy DG, Catani M (2011) Atlasing location, asymmetry and inter-subject variability of white matter tracts in the human brain with MR diffusion tractography. Neuroimage 54:49-59

Toga AW, Thompson PM (2003) Mapping brain asymmetry. Nat Rev Neurosci 4:37-48

Tzourio N, Nkanga-Ngila B, Mazoyer B (1998) Left planum temporale surface correlates with functional dominance during story listening. Neuroreport 9:829-833

Tzourio-Mazoyer N, Petit L, Razafimandimby A, Crivello F, Zago L, Jobard G, Joliot M, Mellet E, Mazoyer B (2010a) Left hemisphere lateralization for language in right-handers is controlled in part by familial sinistrality, manual preference strength, and head size. J Neurosci 30:13314-13318

Tzourio-Mazoyer N, Simon G, Crivello F, Jobard G, Zago L, Perchey G, Hervé PY, Joliot M, Petit L, Mellet E, Mazoyer B (2010b) Effect of familial sinistrality on planum temporale surface and brain tissue asymmetries. Cereb Cortex 20:1476-1485

Tzourio-Mazoyer N, Marie D, Zago L, Jobard G, Perchey G, Leroux G, Mellet E, Joliot M, Crivello F, Petit L, Mazoyer B (2015) Heschl's gyrification pattern is related to speechlistening hemispheric lateralization: FMRI investigation in 281 healthy volunteers. Brain Struct Funct 220:1585-1599

van der Knaap LJ, van der Ham IJ (2011) How does the corpus callosum mediate interhemispheric transfer? A review. Behav Brain Res 223:211-221

Vigneau M, Beaucousin V, Hervé PY, Duffau H, Crivello F, Houdé O, Mazoyer B, TzourioMazoyer N (2006) Meta-analyzing left hemisphere language areas: phonology, semantics, and sentence processing. Neuroimage 30:1414-1432

Vigneau M, Beaucousin V, Hervé PY, Jobard G, Petit L, Crivello F, Mellet E, Zago L, Mazoyer B, Tzourio-Mazoyer N (2011) What is right-hemisphere contribution to phonological, lexicosemantic, and sentence processing? Insights from a meta-analysis. Neuroimage 54:577-593

Vingerhoets G, Alderweireldt AS, Vandemaele P, Cai Q, Van der Haegen L, Brysbaert M, Achten E (2013) Praxis and language are linked: evidence from co-lateralization in individuals with atypical language dominance. Cortex 49:172-183

Warrier C, Wong P, Penhune V, Zatorre R, Parrish T, Abrams D, Kraus N (2009) Relating structure to function: Heschl's gyrus and acoustic processing. J Neurosci 29:61-69

Westbury CF, Zatorre RJ, Evans AC (1999) Quantifying variability in the planum temporale: a probability map. Cereb Cortex 9:392-405 